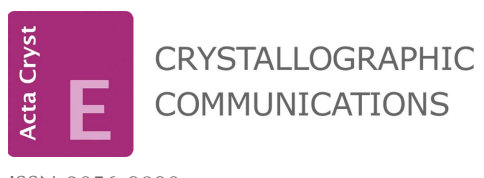

ISSN 2056-9890
Received 9 August 2016

Accepted 22 August 2016

Edited by H. Stoeckli-Evans, University of Neuchâtel, Switzerland

Keywords: crystal structure; julolidine; Schiff base; 8-hydroxyjulolidine-9-carboxaldehyde; p-phenylenediamine; hydrogen bonding; $\mathrm{C}-\mathrm{H} \cdots \pi$ interactions.

CCDC reference: 1500381

Supporting information: this article has supporting information at journals.iucr.org/e

\section{Crystal structure of $9,9^{\prime}-\left\{\left(1 E, 1^{\prime} E\right)\right.$-[1,4-phenyl- enebis(azanylylidene)]bis(methanylylidene)\}- bis(2,3,6,7-tetrahydro- $1 \mathrm{H}, 5 \mathrm{H}$-pyrido[3,2,1-ij]- quinolin-8-ol)}

\author{
Md. Serajul Haque Faizi, ${ }^{a}$ Akram Ali ${ }^{b}$ and Vadim A. Potaskalov ${ }^{c *}$
}

${ }^{\mathbf{a}}$ Department of Chemistry, College of Science, Sultan Qaboos University, PO Box 36 Al-Khod 123, Muscat, Sultanate of,
Oman, ${ }^{\mathbf{b}}$ Department of Chemistry, Indian Institute of Technology Kanpur, Kanpur, UP 208 016, India, and ${ }^{\mathbf{c}}$ Department
of General and Inorganic Chemistry, National Technical University of Ukraine, Kyiv Polytechnic Institute, 37 Prospect
Peremogy, 03056 Kiev, Ukraine. *Correspondence e-mail: potaskalov@xtf.kpi.ua

The whole molecule of the title compound, $\mathrm{C}_{32} \mathrm{H}_{34} \mathrm{~N}_{2} \mathrm{O}_{2}$, is generated by inversion symmetry; the central benzene ring being situated about the crystallographic inversion center. The aromatic ring of the julolidine moiety is inclined to the central benzene ring by $33.70(12)^{\circ}$. There are two intramolecular $\mathrm{O}-\mathrm{H} \cdots \mathrm{N}$ hydrogen bonds in the molecule, generating $S(6)$ ring motifs. The conformation about the $\mathrm{C}=\mathrm{N}$ bonds is $E$. The fused non-aromatic rings of the julolidine moiety adopt half-chair conformations. In the crystal, adjacent molecules are linked by pairs of $\mathrm{C}-\mathrm{H} \cdots \pi$ interactions, forming a ladder-like structure propagating along the $a$-axis direction.

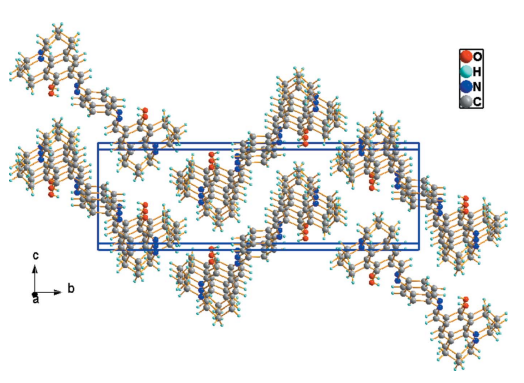

OPEN $\odot$ ACCESS

\section{Chemical context}

8-Hydroxyjulolidine-9-carboxaldehyde is a well-known chromophore used in fluorescence chemosensors; chemosensors with the julolidine moiety are usually soluble in aqueous solutions (Narayanaswamy \& Govindaraju, 2012; Maity et al., 2011; Na et al., 2013; Noh et al., 2013). Compounds containing the julolidine group display chromogenic naked-eye detection of copper, zinc, iron, and aluminium ions as well as fluoride ions (Choi et al., 2015; Wang et al., 2013a,b; Kim et al., 2015; Jo et al., 2015). There are many reports in the literature on 8-hydroxyjulolidine-9-carboxaldehyde-based Schiff bases and their applications as sensors for metal ions (Park et al., 2014; Lee et al., 2014; Kim et al., 2016). Intramolecular $\mathrm{C}-\mathrm{H} \cdots \mathrm{N}$ hydrogen bonds have been observed in a julolidine-derived structure (Barbero et al., 2012). Julolidine dyes exhibiting excited-state intramolecular proton transfer (Nano et al., 2015) and julolidine ring-containing compounds are also fluorescent probes for the measurement of cell-membrane viscosity. The present work is a part of an ongoing structural study of Schiff bases and their utilization in the synthesis of new organic and polynuclear coordination compounds (Faizi \& Sen 2014; Faizi et al., 2016). Recently Choi et al. (2016) have reported on a new chemosensor, similar to the title compound, which is a fluorescent chemosensor for the selective detection of $\mathrm{Zn}^{2+}$ in aqueous solution. This was synthesized by a condensation reaction of 8-hydroxyjulolidine-9-carboxaldehyde with 2-(aminomethyl)benzeneamine in ethanol at room temperature. We report herein on the synthesis and crystal structure of the title julolidine derivative. 
<smiles>Oc1c(/C=N/c2ccc(/N=C/c3cc4c5c(c3O)CCCN5CCC4)cc2)cc2c3c1CCCN3CCC2</smiles>

\section{Structural commentary}

The molecular structure of the title compound is illustrated in Fig. 1. The whole molecule of the title compound is generated by crystallographic inversion symmetry. The conformation about the azomethine $\mathrm{C} 4=\mathrm{N} 1$ bond $[1.285$ (3) $\AA]$ is $E$. The $\mathrm{C} 3-\mathrm{N} 1-\mathrm{C} 4-\mathrm{C} 5$ torsion angle is $172.9(2)^{\circ}$. The molecule is non-planar, with the dihedral angle between the central benzene ring and the aromatic ring of the julolidine moiety being $33.70(12)^{\circ}$. Depending on the tautomers, two types of intramolecular hydrogen bonds are observed in Schiff bases: $\mathrm{O}-\mathrm{H} \cdots \mathrm{N}$ in phenol-imine and $\mathrm{N}-\mathrm{H} \cdots \mathrm{O}$ in keto-amine tautomers. The present analysis shows that the title compound exists in the phenol-imine form (Fig. 1). It exhibits two intramolecular $\quad \mathrm{O} 1-\mathrm{H} 1 A \cdots \mathrm{N} 1 \quad[d(\mathrm{~N} \cdots \mathrm{O}) \quad 2.579(3) \AA]$ hydrogen bonds, which generate $S(6)$ ring motifs (Fig. 1 and Table 1).

\section{Supramolecular features}

In the crystal, adjacent molecules are linked by a pair of $\mathrm{C}-$ $\mathrm{H} \cdots \pi$ interactions (Table 1 and Fig. 2), forming a ladder-like structure propagating along the $a$-axis direction (Fig. 3).

\section{Database survey}

There are very few examples of similar compounds in the literature and, to the best of our knowledge, the new fluor-

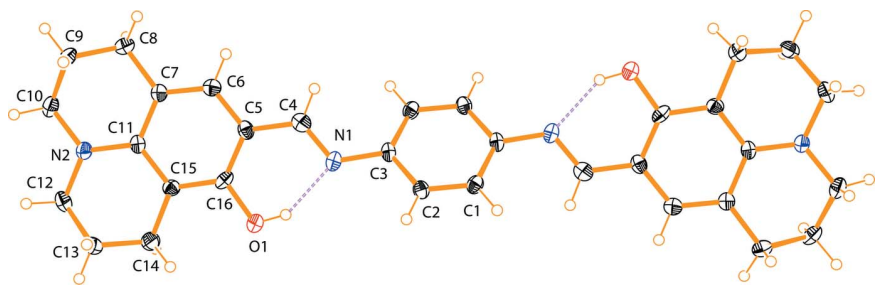

Figure 1

The molecular structure of the title compound, with atom labelling. Displacement ellipsoids are drawn at the $40 \%$ probability level. Unlabelled atoms are generated by the symmetry operation $-x,-y+1$, $-z$. The intramolecular $\mathrm{O}-\mathrm{H} \cdots \mathrm{N}$ hydrogen bonds (see Table 1) are shown as dashed lines.
Table 1

Hydrogen-bond geometry $\left(\AA,^{\circ}\right)$.

$\mathrm{Cg}$ is the centroid of the $\mathrm{C} 5-\mathrm{C} 7 / \mathrm{C} 11 / \mathrm{C} 15 / \mathrm{C} 16$ ring.

\begin{tabular}{lllll}
\hline$D-\mathrm{H} \cdots A$ & $D-\mathrm{H}$ & $\mathrm{H} \cdots A$ & $D \cdots A$ & $D-\mathrm{H} \cdots A$ \\
\hline $\mathrm{O} 1-\mathrm{H} 1 A \cdots \mathrm{N} 1$ & 0.82 & 1.85 & $2.579(3)$ & 148 \\
$\mathrm{C} 10-\mathrm{H} 10 B \cdots C g^{\mathrm{i}}$ & 0.97 & 2.68 & $3.603(3)$ & 160 \\
\hline
\end{tabular}

Symmetry code: (i) $x+1, y, z$.

escent chemosensor for the selective detection of $\mathrm{Zn}^{2+}$ in aqueous solution, mentioned in the Chemical context section (Choi et al., 2016) has not been characterized crystallographically. A search of the Cambridge Structural Database (CSD, Version 5.37, update May 2016; Groom et al., 2016) gave 120 hits for the julolidine moiety. Of these, six have an $\mathrm{OH}$ group in position 8 , and four also have a $\mathrm{C}=\mathrm{N}$ group in position 1 . Of the latter, one compound, viz. 9-\{[(4-chlorophenyl)imino]methyl\}-1,1,7,7-tetramethyl-2,3,6,7-tetrahydro1H,5H-pyrido[3,2,1-ij]quinolin-8-ol (CSD refcode: IGALUZ; Kantar et al., 2013), resembles the title compound and also exists in the phenol-imine form with an intramolecular $\mathrm{O}-$ $\mathrm{H} \cdots \mathrm{N}$ hydrogen bond.

\section{Synthesis and crystallization}

An ethanolic solution of 8-hydroxyjulolidine-9-carboxaldehyde $(100 \mathrm{mg}, 0.46 \mathrm{mmol})$ was added to $p$-phenylenediamine $(25 \mathrm{mg}, 0.23 \mathrm{mmol})$ in absolute ethanol ( $3 \mathrm{ml})$. Two drops of $\mathrm{HCl}$ were added to the reaction solution and it was stirred for $30 \mathrm{~min}$ at room temperature. The resulting yellow precipitate was recovered by filtration, washed several times with small portions of ice-cold EtOH and then with diethyl ether to give $199 \mathrm{mg}(85 \%)$ of the title compound. Crystals suitable for $\mathrm{X}$-ray diffraction analysis were obtained within three days by slow evaporation of a solution in methanol.

\section{Refinement}

Crystal data, data collection and structure refinement details are summarized in Table 2. The $\mathrm{OH}$ and $\mathrm{C}$-bound $\mathrm{H}$ atoms were included in calculated positions and treated as riding atoms: $\mathrm{O}-\mathrm{H}=0.82$ and $\mathrm{C}-\mathrm{H}=0.93-0.97 \AA$, with $U_{\text {iso }}(\mathrm{H})=$ $1.5 U_{\mathrm{eq}}(\mathrm{O})$ and $1.2 U_{\mathrm{eq}}(\mathrm{C})$ for other $\mathrm{H}$ atoms.

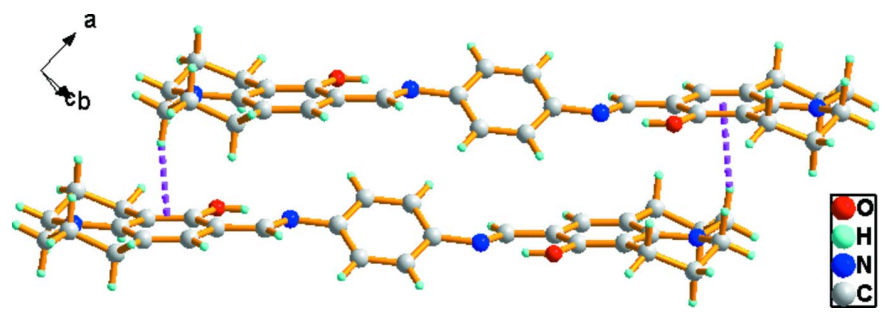

Figure 2

A view of the $\mathrm{C}-\mathrm{H} \cdots \pi$ interactions, shown as dashed lines (see Table 1 ), in the crystal of the title compound. 


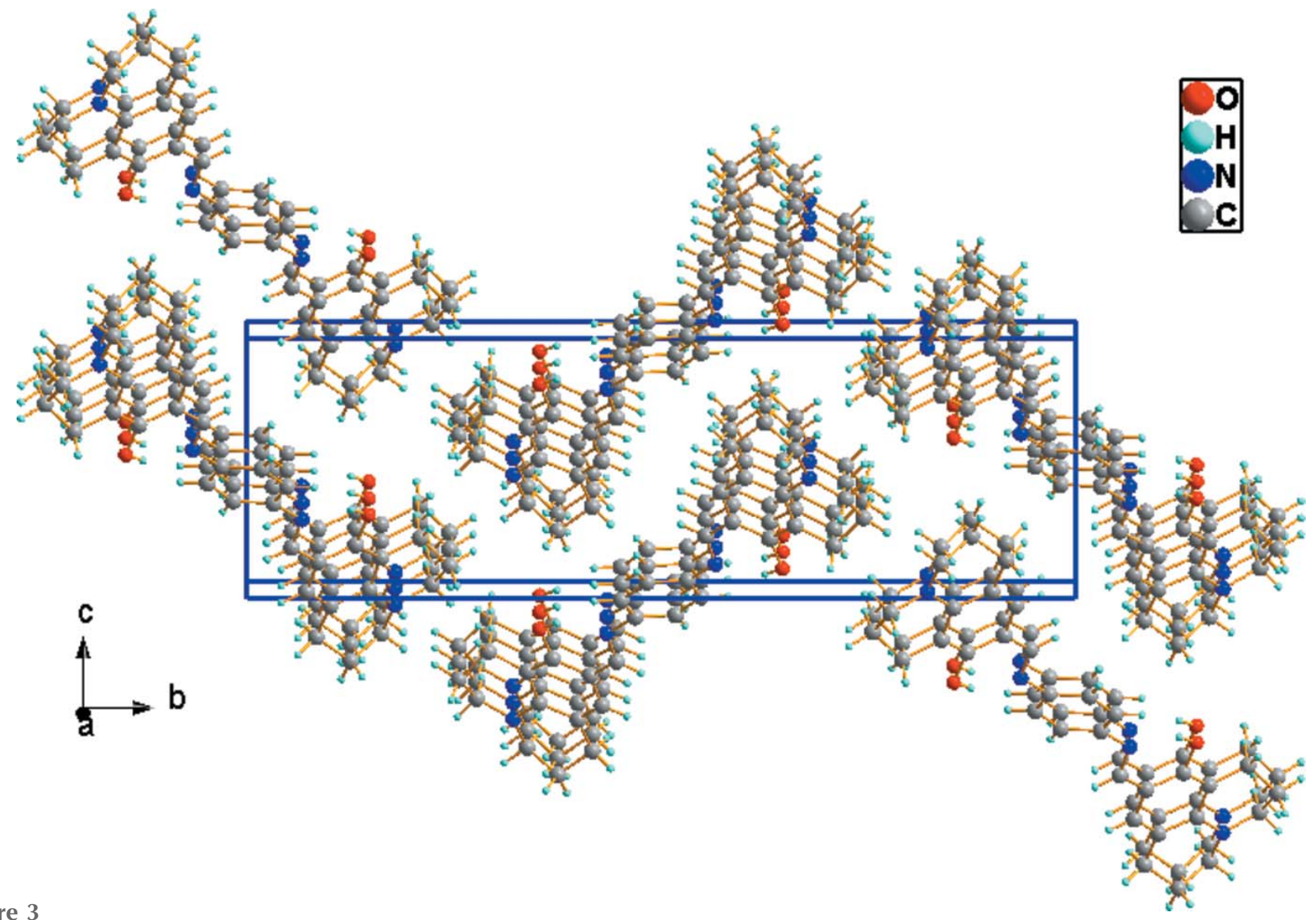

Figure 3

A view along the $a$ axis of the crystal packing of the title compound.

Table 2

Experimental details.

\begin{tabular}{|c|c|}
\hline \multicolumn{2}{|l|}{ Crystal data } \\
\hline Chemical formula & $\mathrm{C}_{32} \mathrm{H}_{34} \mathrm{~N}_{4} \mathrm{O}_{2}$ \\
\hline$M_{\mathrm{r}}$ & 506.63 \\
\hline Crystal system, space group & Monoclinic, $P 2_{1} / c$ \\
\hline Temperature (K) & 100 \\
\hline$a, b, c(\AA)$ & $5.1776(3), 27.9346(17), 8.7893(6)$ \\
\hline$\beta\left(^{\circ}\right)$ & $96.203(2)$ \\
\hline$V\left(\AA^{3}\right)$ & $1263.79(14)$ \\
\hline$Z$ & 2 \\
\hline Radiation type & Mo $K \alpha$ \\
\hline$\mu\left(\mathrm{mm}^{-1}\right)$ & 0.08 \\
\hline Crystal size (mm) & $0.20 \times 0.15 \times 0.12$ \\
\hline \multicolumn{2}{|l|}{ Data collection } \\
\hline Diffractometer & Bruker SMART APEX CCD \\
\hline Absorption correction & $\begin{array}{l}\text { Multi-scan (SADABS; Bruker, } \\
\text { 2003) }\end{array}$ \\
\hline$T_{\min }, T_{\max }$ & $0.783,0.990$ \\
\hline $\begin{array}{l}\text { No. of measured, independent and } \\
\text { observed }[I>2 \sigma(I)] \text { reflections }\end{array}$ & $15125,2243,1469$ \\
\hline$R_{\text {int }}$ & 0.073 \\
\hline$(\sin \theta / \lambda)_{\max }\left(\AA^{-1}\right)$ & 0.596 \\
\hline \multicolumn{2}{|l|}{ Refinement } \\
\hline$R\left[F^{2}>2 \sigma\left(F^{2}\right)\right], w R\left(F^{2}\right), S$ & $0.050,0.127,1.02$ \\
\hline No. of reflections & 2243 \\
\hline No. of parameters & 173 \\
\hline $\mathrm{H}$-atom treatment & $\mathrm{H}$-atom parameters constrained \\
\hline$\Delta \rho_{\max }, \Delta \rho_{\min }\left(\mathrm{e} \AA^{-3}\right)$ & $0.33,-0.22$ \\
\hline
\end{tabular}

Computer programs: SMART and SAINT (Bruker, 2003), SIR97 (Altomare et al., 1999), DIAMOND (Brandenberg \& Putz, 2006), SHELXL97 (Sheldrick, 2008) and PLATON (Spek, 2009).

\section{Acknowledgements}

The authors are grateful to the National Taras Shevchenko University, Department of Chemistry, Volodymyrska str. 64, 01601 Kyiv, Ukraine, for financial support.

\section{References}

Altomare, A., Burla, M. C., Camalli, M., Cascarano, G. L., Giacovazzo, C., Guagliardi, A., Moliterni, A. G. G., Polidori, G. \& Spagna, R. (1999). J. Appl. Cryst. 32, 115-119.

Barbero, N., Barolo, C., Marabello, D., Buscaino, R., Gervasio, G. \& Viscardi, G. (2012). Dyes Pigments, 92, 1177-1183.

Brandenberg, K. \& Putz, H. (2006). DIAMOND. Crystal Impact GbR, Bonn, Germany.

Bruker (2003). SMART, SAINT and SADABS. Bruker AXS Inc., Madison, Wisconsin, USA.

Choi, Y. W., Lee, J. J., You, G. R., Lee, S. Y. \& Kim, C. (2015). RSC Adv. 5, 86463-86472.

Choi, Y. W., You, G. R., Lee, J. J. \& Kim, C. (2016). Inorg. Chem. Commun. 63, 35-38.

Faizi, M. S. H., Gupta, S., Mohan, V. K., Jain, K. V. \& Sen, P. (2016). Sens. Actuators B Chem. 222, 15-20.

Faizi, M. S. H. \& Sen, P. (2014). Acta Cryst. E70, m206-m207.

Groom, C. R., Bruno, I. J., Lightfoot, M. P. \& Ward, S. C. (2016). Acta Cryst. B72, 171-179.

Jo, T. G., Na, Y. J., Lee, J. J., Lee, M. M., Lee, S. Y. \& Kim, C. (2015). New J. Chem. 39, 2580-2587.

Kantar, E. N., Köysal, Y., Akdemir, N., Ağar, A. A. \& Soylu, M. S. (2013). Acta Cryst. E69, o883. 
Kim, Y. S., Lee, J. J., Choi, Y. W., You, G. R., Nguyen, L., Noh, I. \& Kim, C. (2016). Dyes Pigm. 129, 43-53.

Kim, Y. S., Park, G. J., Lee, J. J., Lee, S. Y., Lee, S. Y. \& Kim, C. (2015). RSC Adv. 5, 11229-11239.

Lee, S. A., You, G. R., Choi, Y. W., Jo, H. Y., Kim, A. R., Noh, I., Kim, S.-J., Kim, Y. \& Kim, C. (2014). Dalton Trans. 43, 6650-6659.

Maity, D., Manna, A. K., Karthigeyan, D., Kundu, T. K., Pati, S. K. \& Govindaraju, T. (2011). Chem. Eur. J. 17, 11152-11161.

Na, Y. J., Hwang, I. H., Jo, H. Y., Lee, S. A., Park, G. J. \& Kim, C. (2013). Inorg. Chem. Commun. 35, 342-345.

Nano, A., Gullo, M. P., Ventura, B., Armaroli, N., Barbieri, A. \& Ziessel, R. (2015). Chem. Commun. 51, 3351-3354.
Narayanaswamy, N. \& Govindaraju, T. (2012). Sens. Actuators B Chem. 161, 304-310.

Noh, J. Y., Kim, S., Hwang, I. H., Lee, G. Y., Kang, J., Kim, S. H., Min, J., Park, S., Kim, C. \& Kim, J. (2013). Dyes Pigments, 99, 1016-1021.

Park, G. J., Park, D. Y., Park, K.-M., Kim, Y., Kim, S.-J., Chang, P.-S. \& Kim, C. (2014). Tetrahedron, 70, 7429-7438.

Sheldrick, G. M. (2008). Acta Cryst. A64, 112-122.

Spek, A. L. (2009). Acta Cryst. D65, 148-155.

Wang, L., Li, H. \& Cao, D. (2013a). Sens. Actuators B Chem. 181, 749 755.

Wang, M., Wang, J., Xue, W. \& Wu, A. (2013b). Dyes Pigments, 97, 475-480. 


\section{supporting information}

Acta Cryst. (2016). E72, 1366-1369 [https://doi.org/10.1107/S205698901601344X]

Crystal structure of 9,9'-\{(1E,1'E)-[1,4-phenylenebis(azanylylidene)]bis(methanylylidene)\}bis(2,3,6,7-tetrahydro-1 H,5 H-pyrido[3,2,1-ij]quinolin-8-ol)

\section{Md. Serajul Haque Faizi, Akram Ali and Vadim A. Potaskalov}

Computing details

Data collection: SMART (Bruker, 2003); cell refinement: SAINT (Bruker, 2003); data reduction: SAINT (Bruker, 2003); program(s) used to solve structure: SIR97 (Altomare et al., 1999); program(s) used to refine structure: SHELXL97 (Sheldrick, 2008); molecular graphics: DIAMOND (Brandenberg \& Putz, 2006); software used to prepare material for publication: SHELXL97 (Sheldrick, 2008) and PLATON (Spek, 2009).

9,9'-\{(1E,1'E)-[1,4-Phenylenebis(azanylylidene)]bis(methanylylidene)\}bis(2,3,6,7-tetrahydro-1H,5H-pyrido[3,2,1ij]quinolin-8-ol)

\section{Crystal data}

$\mathrm{C}_{32} \mathrm{H}_{34} \mathrm{~N}_{4} \mathrm{O}_{2}$

$M_{r}=506.63$

Monoclinic, $P 2_{1} / c$

Hall symbol: -P 2ybc

$a=5.1776(3) \AA$

$b=27.9346(17) \AA$

$c=8.7893(6) \AA$

$\beta=96.203(2)^{\circ}$

$V=1263.79(14) \AA^{3}$

$Z=2$

\section{Data collection}

Bruker SMART APEX CCD diffractometer

Radiation source: fine-focus sealed tube Graphite monochromator

/w-scans

Absorption correction: multi-scan

(SADABS; Bruker, 2003)

$T_{\min }=0.783, T_{\max }=0.990$

Refinement

Refinement on $F^{2}$

Least-squares matrix: full

$R\left[F^{2}>2 \sigma\left(F^{2}\right)\right]=0.050$

$w R\left(F^{2}\right)=0.127$

$S=1.02$

2243 reflections

173 parameters

0 restraints
$F(000)=540$

$D_{\mathrm{x}}=1.331 \mathrm{Mg} \mathrm{m}^{-3}$

Mo $K \alpha$ radiation, $\lambda=0.71073 \AA$

Cell parameters from 3371 reflections

$\theta=2.4-26.5^{\circ}$

$\mu=0.08 \mathrm{~mm}^{-1}$

$T=100 \mathrm{~K}$

Block, yellow

$0.20 \times 0.15 \times 0.12 \mathrm{~mm}$

15125 measured reflections

2243 independent reflections

1469 reflections with $I>2 \sigma(I)$

$R_{\text {int }}=0.073$

$\theta_{\max }=25.0^{\circ}, \theta_{\min }=2.8^{\circ}$

$h=-6 \rightarrow 6$

$k=-33 \rightarrow 33$

$l=-10 \rightarrow 10$

Primary atom site location: structure-invariant direct methods

Secondary atom site location: difference Fourier map

Hydrogen site location: inferred from neighbouring sites

$\mathrm{H}$-atom parameters constrained 
$w=1 /\left[\sigma^{2}\left(F_{\mathrm{o}}^{2}\right)+(0.0483 P)^{2}+0.7868 P\right]$

where $P=\left(F_{\mathrm{o}}^{2}+2 F_{\mathrm{c}}{ }^{2}\right) / 3$

$(\Delta / \sigma)_{\max }<0.001$

$$
\Delta \rho_{\max }=0.33 \text { e } \AA^{-3}
$$

\section{Special details}

Geometry. All esds (except the esd in the dihedral angle between two 1.s. planes) are estimated using the full covariance matrix. The cell esds are taken into account individually in the estimation of esds in distances, angles and torsion angles; correlations between esds in cell parameters are only used when they are defined by crystal symmetry. An approximate (isotropic) treatment of cell esds is used for estimating esds involving l.s. planes.

Refinement. Refinement of $\mathrm{F}^{2}$ against ALL reflections. The weighted R-factor $\mathrm{wR}$ and goodness of fit $\mathrm{S}$ are based on $\mathrm{F}^{2}$, conventional R-factors $R$ are based on $F$, with $F$ set to zero for negative $\mathrm{F}^{2}$. The threshold expression of $\mathrm{F}^{2}>2 \operatorname{sigma}\left(\mathrm{F}^{2}\right)$ is used only for calculating R-factors(gt) etc. and is not relevant to the choice of reflections for refinement. R-factors based on $\mathrm{F}^{2}$ are statistically about twice as large as those based on F, and R- factors based on ALL data will be even larger.

Fractional atomic coordinates and isotropic or equivalent isotropic displacement parameters $\left(\AA^{2}\right)$

\begin{tabular}{|c|c|c|c|c|}
\hline & $x$ & $y$ & $z$ & $U_{\text {iso }} * / U_{\text {eq }}$ \\
\hline $\mathrm{O} 1$ & $0.5852(3)$ & $0.64668(6)$ & $0.15270(19)$ & $0.0327(5)$ \\
\hline H1A & 0.4785 & 0.6259 & 0.1246 & $0.049 *$ \\
\hline N2 & $1.2746(4)$ & $0.67853(7)$ & $0.5427(2)$ & $0.0250(5)$ \\
\hline N1 & 0.3470 & $0.56564(7)$ & $0.1548(2)$ & $0.0259(5)$ \\
\hline $\mathrm{C} 3$ & $0.1732(4)$ & $0.53146(8)$ & $0.0816(3)$ & $0.0216(6)$ \\
\hline $\mathrm{C} 11$ & $1.0954(4)$ & $0.64728(8)$ & $0.4704(3)$ & $0.0208(6)$ \\
\hline $\mathrm{C} 7$ & 1.0677 (4) & $0.60049(8)$ & $0.5312(3)$ & $0.0212(6)$ \\
\hline $\mathrm{C} 1$ & $-0.2269(4)$ & $0.51736(9)$ & $-0.0764(3)$ & $0.0249(6)$ \\
\hline H1 & -0.3807 & 0.5292 & -0.1271 & $0.030^{*}$ \\
\hline $\mathrm{C} 15$ & $0.9341(4)$ & $0.66163(8)$ & 0.3388 & $0.0229(6)$ \\
\hline C16 & $0.7394(5)$ & $0.63101(9)$ & 0.2775 & $0.0250(6)$ \\
\hline C6 & $0.8730(4)$ & $0.57163(9)$ & 0.4650 & $0.0245(6)$ \\
\hline H6 & 0.8546 & 0.5412 & 0.5055 & $0.029 *$ \\
\hline $\mathrm{C} 2$ & $-0.0546(4)$ & $0.54825(9)$ & 0.0030 & $0.0240(6)$ \\
\hline $\mathrm{H} 2$ & -0.0918 & 0.5808 & 0.0039 & $0.029 *$ \\
\hline $\mathrm{C} 5$ & $0.7015(5)$ & $0.58567(8)$ & 0.3400 & $0.0241(6)$ \\
\hline $\mathrm{C} 4$ & $0.5029(5)$ & $0.55395(9)$ & $0.2728(3)$ & $0.0277(6)$ \\
\hline $\mathrm{H} 4$ & 0.4865 & 0.5239 & 0.3161 & $0.033^{*}$ \\
\hline $\mathrm{C} 8$ & $1.2531(5)$ & $0.58325(9)$ & $0.6635(3)$ & $0.0277(6)$ \\
\hline H8A & 1.3963 & 0.5663 & 0.6250 & $0.033^{*}$ \\
\hline H8B & 1.1643 & 0.5611 & 0.7251 & $0.033^{*}$ \\
\hline $\mathrm{C} 12$ & $1.3361(5)$ & $0.72291(9)$ & $0.4669(3)$ & $0.0305(6)$ \\
\hline $\mathrm{H} 12 \mathrm{~A}$ & 1.4648 & 0.7165 & 0.3971 & $0.037^{*}$ \\
\hline H12B & 1.4101 & 0.7456 & 0.5429 & $0.037 *$ \\
\hline $\mathrm{C} 10$ & $1.4682(5)$ & $0.66195(9)$ & $0.6634(3)$ & $0.0305(6)$ \\
\hline $\mathrm{H} 10 \mathrm{~A}$ & 1.5310 & 0.6889 & 0.7264 & $0.037^{*}$ \\
\hline $\mathrm{H} 10 \mathrm{~B}$ & 1.6144 & 0.6484 & 0.6181 & $0.037^{*}$ \\
\hline $\mathrm{C} 14$ & $0.9740(5)$ & $0.70887(9)$ & $0.2652(3)$ & $0.0307(6)$ \\
\hline $\mathrm{H} 14 \mathrm{~A}$ & 0.8078 & 0.7212 & 0.2203 & $0.037^{*}$ \\
\hline H14B & 1.0841 & 0.7046 & 0.1837 & $0.037^{*}$ \\
\hline $\mathrm{C} 13$ & $1.0976(5)$ & $0.74444(9)$ & 0.3793 & $0.0313(6)$ \\
\hline H13A & 1.1453 & 0.7730 & 0.3264 & $0.038^{*}$ \\
\hline
\end{tabular}


supporting information

\begin{tabular}{lllll} 
H13B & 0.9744 & 0.7535 & 0.4499 & $0.038^{*}$ \\
C9 & $1.3577(5)$ & $0.62498(9)$ & $0.7617(3)$ & $0.0309(6)$ \\
H9A & 1.2190 & 0.6390 & 0.8130 & $0.037^{*}$ \\
H9B & 1.4917 & 0.6138 & 0.8393 & $0.037^{*}$ \\
\hline
\end{tabular}

Atomic displacement parameters $\left(\AA^{2}\right)$

\begin{tabular}{lllllll}
\hline & $U^{11}$ & $U^{22}$ & $U^{33}$ & $U^{12}$ & $U^{13}$ & $U^{23}$ \\
\hline O1 & $0.0348(11)$ & $0.0320(11)$ & $0.0288(10)$ & $-0.0051(8)$ & $-0.0072(9)$ & $0.0013(8)$ \\
N2 & $0.0219(11)$ & $0.0267(12)$ & $0.0257(12)$ & $-0.0030(10)$ & $-0.0006(9)$ & $-0.0002(9)$ \\
N1 & $0.0213(11)$ & $0.0324(13)$ & $0.0233(11)$ & $-0.0016(10)$ & $-0.0003(10)$ & $-0.0044(10)$ \\
C3 & $0.0198(12)$ & $0.0261(13)$ & $0.0198(13)$ & $-0.0057(11)$ & $0.0060(11)$ & $-0.0059(11)$ \\
C11 & $0.0174(12)$ & $0.0239(14)$ & $0.0219(13)$ & $-0.0019(10)$ & $0.0057(11)$ & $-0.0050(10)$ \\
C7 & $0.0213(13)$ & $0.0239(14)$ & $0.0192(13)$ & $0.0009(11)$ & $0.0061(11)$ & $-0.0041(11)$ \\
C1 & $0.0187(13)$ & $0.0316(15)$ & $0.0241(14)$ & $0.0004(11)$ & $0.0016(11)$ & $-0.0006(11)$ \\
C15 & $0.0248(13)$ & $0.0244(13)$ & $0.0202(13)$ & $-0.0008(11)$ & $0.0049(11)$ & $0.0019(11)$ \\
C16 & $0.0229(13)$ & $0.0350(15)$ & $0.0165(12)$ & $0.0061(12)$ & $-0.0010(11)$ & $-0.0004(11)$ \\
C6 & $0.0273(14)$ & $0.0252(14)$ & $0.0217(13)$ & $0.0020(11)$ & $0.0065(11)$ & $-0.0004(11)$ \\
C2 & $0.0239(13)$ & $0.0233(14)$ & $0.0251(14)$ & $-0.0017(11)$ & $0.0042(11)$ & $-0.0038(11)$ \\
C5 & $0.0263(14)$ & $0.0236(14)$ & $0.0234(14)$ & $-0.0039(11)$ & $0.0070(12)$ & $-0.0052(11)$ \\
C4 & $0.0312(14)$ & $0.0263(14)$ & $0.0270(14)$ & $0.0006(12)$ & $0.0098(12)$ & $-0.0024(12)$ \\
C8 & $0.0297(15)$ & $0.0305(15)$ & $0.0229(14)$ & $0.0056(12)$ & $0.0030(12)$ & $0.0031(11)$ \\
C12 & $0.0275(14)$ & $0.0250(14)$ & $0.0394(16)$ & $-0.0064(12)$ & $0.0048(12)$ & $-0.0054(12)$ \\
C10 & $0.0253(13)$ & $0.0350(15)$ & $0.0296(15)$ & $0.0023(12)$ & $-0.0043(12)$ & $-0.0082(12)$ \\
C14 & $0.0289(14)$ & $0.0323(15)$ & $0.0305(15)$ & $-0.0034(12)$ & $0.0015(12)$ & $0.0033(12)$ \\
C13 & $0.0356(15)$ & $0.0255(14)$ & $0.0329(15)$ & $-0.0034(12)$ & $0.0047(13)$ & $0.0045(12)$ \\
C9 & $0.0292(14)$ & $0.0384(16)$ & $0.0233(14)$ & $0.0096(13)$ & $-0.0056(11)$ & $-0.0031(12)$ \\
& & & & & &
\end{tabular}

Geometric parameters $\left(\AA,^{\circ}\right)$

\begin{tabular}{llll}
\hline $\mathrm{O} 1-\mathrm{C} 16$ & $1.358(3)$ & $\mathrm{C} 6-\mathrm{H} 6$ & 0.9300 \\
$\mathrm{O} 1-\mathrm{H} 1 \mathrm{~A}$ & 0.8200 & $\mathrm{C} 2-\mathrm{H} 2$ & 0.9300 \\
$\mathrm{~N} 2-\mathrm{C} 11$ & $1.378(3)$ & $\mathrm{C} 5-\mathrm{C} 4$ & $1.435(3)$ \\
$\mathrm{N} 2-\mathrm{C} 10$ & $1.454(3)$ & $\mathrm{C} 4-\mathrm{H} 4$ & 0.9300 \\
$\mathrm{~N} 2-\mathrm{C} 12$ & $1.459(3)$ & $\mathrm{C} 8-\mathrm{C} 9$ & $1.515(3)$ \\
$\mathrm{N} 1-\mathrm{C} 4$ & $1.285(3)$ & $\mathrm{C} 8-\mathrm{H} 8 \mathrm{~A}$ & 0.9700 \\
$\mathrm{~N} 1-\mathrm{C} 3$ & $1.418(3)$ & $\mathrm{C} 8-\mathrm{H} 8 \mathrm{~B}$ & 0.9700 \\
$\mathrm{C} 3-\mathrm{C} 2$ & $1.383(3)$ & $\mathrm{C} 12-\mathrm{C} 13$ & $1.508(3)$ \\
$\mathrm{C} 3-\mathrm{C} 1^{\mathrm{i}}$ & $1.394(3)$ & $\mathrm{C} 12-\mathrm{H} 12 \mathrm{~A}$ & 0.9700 \\
$\mathrm{C} 11-\mathrm{C} 15$ & $1.410(3)$ & $\mathrm{C} 12-\mathrm{H} 12 \mathrm{~B}$ & 0.9700 \\
$\mathrm{C} 11-\mathrm{C} 7$ & $1.425(3)$ & $\mathrm{C} 10-\mathrm{C} 9$ & $1.499(4)$ \\
$\mathrm{C} 7-\mathrm{C} 6$ & $1.370(3)$ & $\mathrm{C} 10-\mathrm{H} 10 \mathrm{~A}$ & 0.9700 \\
$\mathrm{C} 7-\mathrm{C} 8$ & $1.505(3)$ & $\mathrm{C} 10-\mathrm{H} 10 \mathrm{~B}$ & 0.9700 \\
$\mathrm{C} 1-\mathrm{C} 2$ & $1.376(3)$ & $\mathrm{C} 14-\mathrm{C} 13$ & $1.505(3)$ \\
$\mathrm{C} 1-\mathrm{C} 3$ & $\mathrm{C} 14-\mathrm{H} 14 \mathrm{~A}$ & 0.9700 \\
$\mathrm{C} 1-\mathrm{H} 1$ & $1.394(3)$ & $\mathrm{C} 14-\mathrm{H} 14 \mathrm{~B}$ & 0.9700 \\
$\mathrm{C} 15-\mathrm{C} 16$ & 0.9300 & $\mathrm{C} 13-\mathrm{H} 13 \mathrm{~A}$ & 0.9700 \\
$\mathrm{C} 15-\mathrm{C} 14$ & $1.386(3)$ & $\mathrm{C} 13-\mathrm{H} 13 \mathrm{~B}$ & 0.9700
\end{tabular}




\begin{tabular}{|c|c|c|c|}
\hline $\mathrm{C} 16-\mathrm{C} 5$ & $1.403(3)$ & $\mathrm{C} 9-\mathrm{H} 9 \mathrm{~A}$ & 0.9700 \\
\hline $\mathrm{C} 6-\mathrm{C} 5$ & $1.392(3)$ & $\mathrm{C} 9-\mathrm{H} 9 \mathrm{~B}$ & 0.9700 \\
\hline $\mathrm{C} 16-\mathrm{O} 1-\mathrm{H} 1 \mathrm{~A}$ & 109.5 & $\mathrm{C} 7-\mathrm{C} 8-\mathrm{H} 8 \mathrm{~A}$ & 109.5 \\
\hline $\mathrm{C} 11-\mathrm{N} 2-\mathrm{C} 10$ & $120.75(19)$ & $\mathrm{C} 9-\mathrm{C} 8-\mathrm{H} 8 \mathrm{~A}$ & 109.5 \\
\hline $\mathrm{C} 11-\mathrm{N} 2-\mathrm{C} 12$ & $119.8(2)$ & $\mathrm{C} 7-\mathrm{C} 8-\mathrm{H} 8 \mathrm{~B}$ & 109.5 \\
\hline $\mathrm{C} 10-\mathrm{N} 2-\mathrm{C} 12$ & $115.88(19)$ & $\mathrm{C} 9-\mathrm{C} 8-\mathrm{H} 8 \mathrm{~B}$ & 109.5 \\
\hline $\mathrm{C} 4-\mathrm{N} 1-\mathrm{C} 3$ & $120.5(2)$ & $\mathrm{H} 8 \mathrm{~A}-\mathrm{C} 8-\mathrm{H} 8 \mathrm{~B}$ & 108.1 \\
\hline $\mathrm{C} 2-\mathrm{C} 3-\mathrm{C}^{\mathrm{i}}$ & $118.6(2)$ & $\mathrm{N} 2-\mathrm{C} 12-\mathrm{C} 13$ & $111.4(2)$ \\
\hline $\mathrm{C} 2-\mathrm{C} 3-\mathrm{N} 1$ & $117.6(2)$ & $\mathrm{N} 2-\mathrm{C} 12-\mathrm{H} 12 \mathrm{~A}$ & 109.3 \\
\hline $\mathrm{C} 1-\mathrm{C} 3-\mathrm{N} 1$ & $123.7(2)$ & $\mathrm{C} 13-\mathrm{C} 12-\mathrm{H} 12 \mathrm{~A}$ & 109.3 \\
\hline $\mathrm{N} 2-\mathrm{C} 11-\mathrm{C} 15$ & $120.5(2)$ & $\mathrm{N} 2-\mathrm{C} 12-\mathrm{H} 12 \mathrm{~B}$ & 109.3 \\
\hline $\mathrm{N} 2-\mathrm{C} 11-\mathrm{C} 7$ & $119.9(2)$ & $\mathrm{C} 13-\mathrm{C} 12-\mathrm{H} 12 \mathrm{~B}$ & 109.3 \\
\hline $\mathrm{C} 15-\mathrm{C} 11-\mathrm{C} 7$ & $119.6(2)$ & $\mathrm{H} 12 \mathrm{~A}-\mathrm{C} 12-\mathrm{H} 12 \mathrm{~B}$ & 108.0 \\
\hline $\mathrm{C} 6-\mathrm{C} 7-\mathrm{C} 11$ & $118.7(2)$ & $\mathrm{N} 2-\mathrm{C} 10-\mathrm{C} 9$ & $111.4(2)$ \\
\hline $\mathrm{C} 6-\mathrm{C} 7-\mathrm{C} 8$ & $121.2(2)$ & $\mathrm{N} 2-\mathrm{C} 10-\mathrm{H} 10 \mathrm{~A}$ & 109.4 \\
\hline $\mathrm{C} 11-\mathrm{C} 7-\mathrm{C} 8$ & $120.1(2)$ & $\mathrm{C} 9-\mathrm{C} 10-\mathrm{H} 10 \mathrm{~A}$ & 109.4 \\
\hline $\mathrm{C} 2-\mathrm{C} 1-\mathrm{C} 3^{\mathrm{i}}$ & $120.6(2)$ & $\mathrm{N} 2-\mathrm{C} 10-\mathrm{H} 10 \mathrm{~B}$ & 109.4 \\
\hline $\mathrm{C} 2-\mathrm{C} 1-\mathrm{H} 1$ & 119.7 & $\mathrm{C} 9-\mathrm{C} 10-\mathrm{H} 10 \mathrm{~B}$ & 109.4 \\
\hline 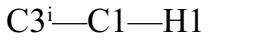 & 119.7 & $\mathrm{H} 10 \mathrm{~A}-\mathrm{C} 10-\mathrm{H} 10 \mathrm{~B}$ & 108.0 \\
\hline $\mathrm{C} 16-\mathrm{C} 15-\mathrm{C} 11$ & $119.0(2)$ & $\mathrm{C} 15-\mathrm{C} 14-\mathrm{C} 13$ & $111.3(2)$ \\
\hline $\mathrm{C} 16-\mathrm{C} 15-\mathrm{C} 14$ & $120.4(2)$ & $\mathrm{C} 15-\mathrm{C} 14-\mathrm{H} 14 \mathrm{~A}$ & 109.4 \\
\hline $\mathrm{C} 11-\mathrm{C} 15-\mathrm{C} 14$ & $120.6(2)$ & $\mathrm{C} 13-\mathrm{C} 14-\mathrm{H} 14 \mathrm{~A}$ & 109.4 \\
\hline $\mathrm{O} 1-\mathrm{C} 16-\mathrm{C} 15$ & $117.0(2)$ & $\mathrm{C} 15-\mathrm{C} 14-\mathrm{H} 14 \mathrm{~B}$ & 109.4 \\
\hline $\mathrm{O} 1-\mathrm{C} 16-\mathrm{C} 5$ & $120.8(2)$ & $\mathrm{C} 13-\mathrm{C} 14-\mathrm{H} 14 \mathrm{~B}$ & 109.4 \\
\hline $\mathrm{C} 15-\mathrm{C} 16-\mathrm{C} 5$ & $122.1(2)$ & $\mathrm{H} 14 \mathrm{~A}-\mathrm{C} 14-\mathrm{H} 14 \mathrm{~B}$ & 108.0 \\
\hline $\mathrm{C} 7-\mathrm{C} 6-\mathrm{C} 5$ & $123.1(2)$ & $\mathrm{C} 12-\mathrm{C} 13-\mathrm{C} 14$ & $110.0(2)$ \\
\hline $\mathrm{C} 7-\mathrm{C} 6-\mathrm{H} 6$ & 118.4 & $\mathrm{C} 12-\mathrm{C} 13-\mathrm{H} 13 \mathrm{~A}$ & 109.7 \\
\hline $\mathrm{C} 5-\mathrm{C} 6-\mathrm{H} 6$ & 118.4 & $\mathrm{C} 14-\mathrm{C} 13-\mathrm{H} 13 \mathrm{~A}$ & 109.7 \\
\hline $\mathrm{C} 1-\mathrm{C} 2-\mathrm{C} 3$ & $120.8(2)$ & $\mathrm{C} 12-\mathrm{C} 13-\mathrm{H} 13 \mathrm{~B}$ & 109.7 \\
\hline $\mathrm{C} 1-\mathrm{C} 2-\mathrm{H} 2$ & 119.6 & $\mathrm{C} 14-\mathrm{C} 13-\mathrm{H} 13 \mathrm{~B}$ & 109.7 \\
\hline $\mathrm{C} 3-\mathrm{C} 2-\mathrm{H} 2$ & 119.6 & $\mathrm{H} 13 \mathrm{~A}-\mathrm{C} 13-\mathrm{H} 13 \mathrm{~B}$ & 108.2 \\
\hline $\mathrm{C} 6-\mathrm{C} 5-\mathrm{C} 16$ & $117.3(2)$ & $\mathrm{C} 10-\mathrm{C} 9-\mathrm{C} 8$ & $109.7(2)$ \\
\hline $\mathrm{C} 6-\mathrm{C} 5-\mathrm{C} 4$ & $121.2(2)$ & $\mathrm{C} 10-\mathrm{C} 9-\mathrm{H} 9 \mathrm{~A}$ & 109.7 \\
\hline $\mathrm{C} 16-\mathrm{C} 5-\mathrm{C} 4$ & $121.4(2)$ & $\mathrm{C} 8-\mathrm{C} 9-\mathrm{H} 9 \mathrm{~A}$ & 109.7 \\
\hline $\mathrm{N} 1-\mathrm{C} 4-\mathrm{C} 5$ & $122.3(2)$ & $\mathrm{C} 10-\mathrm{C} 9-\mathrm{H} 9 \mathrm{~B}$ & 109.7 \\
\hline $\mathrm{N} 1-\mathrm{C} 4-\mathrm{H} 4$ & 118.8 & $\mathrm{C} 8-\mathrm{C} 9-\mathrm{H} 9 \mathrm{~B}$ & 109.7 \\
\hline $\mathrm{C} 5-\mathrm{C} 4-\mathrm{H} 4$ & 118.8 & $\mathrm{H} 9 \mathrm{~A}-\mathrm{C} 9-\mathrm{H} 9 \mathrm{~B}$ & 108.2 \\
\hline $\mathrm{C} 7-\mathrm{C} 8-\mathrm{C} 9$ & $110.7(2)$ & & \\
\hline
\end{tabular}

Symmetry code: (i) $-x,-y+1,-z$.

Hydrogen-bond geometry $\left(\AA,{ }^{\circ}\right)$

$\mathrm{Cg}$ is the centroid of the $\mathrm{C} 5-\mathrm{C} 7 / \mathrm{C} 11 / \mathrm{C} 15 / \mathrm{C} 16$ ring.

\begin{tabular}{lllll}
\hline$D-\mathrm{H} \cdots A$ & $D-\mathrm{H}$ & $\mathrm{H} \cdots A$ & $D \cdots A$ & $D-\mathrm{H} \cdots A$ \\
\hline $\mathrm{O} 1-\mathrm{H} 1 A^{\cdots} \cdots \mathrm{N} 1$ & 0.82 & 1.85 & $2.579(3)$ & 148
\end{tabular}


Symmetry code: (ii) $x+1, y, z$. 